\title{
Research on Channel Estimation of Power Line Communication System under Impulse Interference
}

\author{
Cong Zhang*, Hao Chen
}

Tongji Zhejiang College, Zhejiang 314000, China. E-mail: 240790180@qq.com

\begin{abstract}
The power line communication system is transmitted by the original power line, which has the advantages of low transmission cost and wide range of power line wiring, but it has high research value due to the disadvantages of bad transmission environment and large interference. In this paper, the problem of channel estimation in power line communication system under impulse interference is analyzed objectively, and the improvement measures in its transmission process are put forward.
\end{abstract}

Keywords: Power Line Communication; Transmission Process; Affected by Interference; Improvement Measures

\section{Development status of power line communication}

As early as 1838, an Englishman named Edward Davy had put forward the power line communication scheme. In addition, he also filed a patent in 1897. So far, power line communication began to research and develop in foreign countries. Until the end of the 20th century, China began to put forward the research on power line communication system. Although it started nearly a hundred years later than foreign countries, power line communication technology developed rapidly in China.

Its rapid development is mainly attributed to two aspects. One is the construction of national power grid. With the progress of social science and technology and the rapid development of economy in the 21 st century, the concept of intelligence is constantly deepening into life. The national power grid is also constantly implementing the construction of smart grid, while the power communication system relies on the construction of national power grid to achieve rapid development, using power line communication to realize intelligence energy has promoted social progress. Second, power line communication has the feature of low cost. Power line communication relies on the national grid as the carrier. As long as there is a national grid, there can be power line communication. There is no need to build communication equipment or communication transfer equipment, which greatly reduces the cost of construction. In addition, the national grid is widely distributed in many mountainous areas, so there is power line communication. It can be said that the letter can produce higher economic benefits throughout the country. But because the power line communication is very easy to be interfered, its transmission environment is also bad, which leads to the relative instability and some problems of power line communication, so it is of great significance to solve the related problems of power line communication.

\section{Impact of system pulse interference}

In power line communication system, the transmission mode of OFDM multi frequency multiplexing is commonly used, because of its strong anti-interference ability, so this paper is also based on power line communication under OFDM, because power line communication system is very vulnerable to interference, and the quality of power line communication is seriously affected by pulse interference, which can better suppress the interference of pulse can help

This is an open-access article distributed under the terms of the Creative Commons Attribution Non-Commercial License (http://creativecommons. org/licenses/by-nc/4.0/), which permits unrestricted non-commercial use, distribution, and reproduction in any medium, provided the original work is properly cited. 
to improve the quality of power line communication. Under the interference of pulse, it mainly affects the following two aspects.

\subsection{Impact on bit error rate under pulse interference}

Power line communication system is built on the basis of electric power and transmitted through power line. In the process of transmission belt, there is no point-to-point transmission like telephone line and optical fiber, and there is very small external pulse interference when transferring from base station to users. Power line communication system is sent out from the transmitter through electric power, and a large number of power equipment, such as electricity, are transferred along the way line, energy consuming equipment, etc. Therefore, the communication channel has more pulse interference in the transmission process. These additional pulse interferences will seriously affect the transmission of signals. The original 01 sent by the sender may not be the original 01 received at the receiver after transmission through the power line communication system, but become other signals. When the sender sends a long series of signals, the wrong codewords will become more and more, and the corresponding error codewords will continue to become more and more after transmission through the power line. The strength of pulse interference will have a serious impact on the bit error rate. The higher the strong bit error rate of interference, the more unstable the transmission.

\subsection{Effect on frequency selectivity under pulse interference}

Due to the multi-path characteristics of the channel (described in the physical structure below), the frequency components of the power line communication system passing through the signal will be inconsistent under the pulse interference, because when the signal bandwidth is larger than the related channel bandwidth when the symbol rate is high, the signal frequency after passing through the transmission is inconsistent, resulting in frequency selective fading and waveform distortion. It will cause inter code interference, because the interference with pulse during power line transmission will have a great impact on the quality of communication.

\section{Improvement measures during transmission}

The power line communication system has the advantages of low operating equipment cost and high economic benefits, but at the same time, it also has the disadvantages brought by the power line. In the transmission process, it will inevitably be subject to external interference, resulting in the low quality of communication transmission, so that its practicability is greatly reduced. Therefore, it is particularly important for the improvement measures in the transmission process. Some specific measures. It will greatly reduce the errors in the transmission process, improve the quality of communication, so that it can be used in your society.

\subsection{Application of physical structure in power line communication}

Because the power line communication system is based on the power line, it depends on the original power system, and takes the power system as the transmission medium, so many physical characteristics in the power line communication system will have a huge impact on the system communication, such as the size of resistance in the power line, the transmission frequency, and the connection mode will affect the communication quality in the physical level quantity. Firstly, in the process of power line transmission, the impedance is constantly changing, which means the attenuation of the channel will be different, so the attenuation of the channel is time-varying. Secondly, the frequency in the transmission process of the original power line has a huge impact on the power line communication. When the transmission frequency is large, the corresponding attenuation will be larger, and the attenuation will be smaller when the frequency is small. The corresponding reduction of the transmission frequency is of great significance for the transmission of power line communication. In addition, the signal may reflect and fold in the transmission process when the power line is transmitted. In the process of power line transmission, it also has multi-path characteristics. As a result, the communication quality of power line communication system is not high. So we need to pay attention to the application of physical structure in the process of power line communication.

\subsection{Application of coding in power line communication}

The power line communication system will generate wrong symbols under the interference of pulses. The wrong symbols will affect the quality of communication, resulting in the difference of information between the sender and 
the receiver in the transmission process. Because in the process of power line communication, the use of coding is adopted, and the error through pulse interference is usually sudden, while the noise interference of the channel itself is used. The signal is a random error. No matter what kind of forward error correction coding technology is used, some error correction codes are added to the information sent by the original transmitting end. These codes are called error correction codes. The error correction codes are arranged in a certain rule with the original information. When the receiving end finds the error when receiving the signal, the established rules of error correction codes can be used for error correction. This coding method greatly reduces the error caused by interference, greatly increases the quality of power line communication, and improves the feasibility and usability of power line communication system.

\subsection{Application of spread spectrum in power communication}

In order to improve the quality of power line communication and spread spectrum, Shannon Formula, a famous mathematician and founder of information theory, has been put forward. In the formula, it is pointed out that the transmission rate of information is proportional to the product of bandwidth and signal-to-noise ratio. On the premise of the transmission rate of information, properly increasing the bandwidth of information transmission will reduce the transmission process. The requirement of signal-to-noise ratio, that is to say, expanding the bandwidth of information as much as possible makes the anti-interference tolerance greatly improved, the quality of information in the transmission process of power line will also be improved, and the errors in the corresponding transmission process will be reduced. In addition to the shortage of spectrum resources in today's society, all countries are competing for better spectrum resources, while the frequency of the transmitter of the spread spectrum technology is small, which is easy to be reused in the same area, and has high utilization value.

\section{Summary and proposal}

To sum up, power line communication has unique advantages in convenience and economic value. On the basis of the vigorous construction of the state grid in the early stage, it paves the way for power line communication without the cost of transmission equipment, which greatly reduces the transmission cost. In addition, the construction of the power grid enables power line communication to reach all parts of the country, so that some mountainous areas and other places are greatly improved. At the same time, the power line communication also has positive and obvious disadvantages, which is very easy to be interfered. Under the pulse interference, it will have a serious impact on the channel and affect the transmission quality. When using the power line communication system for transmission, we should first consider from the physical level, consider the channel impedance, transmission frequency, transmission distance and other factors that are easy to change in the transmission process, reduce the error rate in the process of information transmission as much as possible, and improve the quality of system transmission. When the quality of external transmission is not high, we should use additional technology for correction, such as coding technology to improve the quality and reliability of communication.

\section{References}

1. Zhang J, Zheng B. Overview of pilot-aided channel estimation in OFDM. Journal of China Institute of Communications 2003; 24(11): 116-124.

2. Qi S. Guodian Communication Center successfully opened the PLC test network developed by ourselves in China (in Chinese). Power System Communication 2002; (6): 54-55.

3. Zhang G, Xiong X. Interference characteristics and suppression techniques in power line communication. Telecommunications Science 2016; 32(2): 182-188. doi: 10.11959/j.issn.1000-0801.2016041. 